\title{
Blind Equalization of Time Errors in a Time Interleaved ADC System
}

\author{
Jonas Elbornsson, Fredrik Gustafsson, Jan-Erik Eklund
}

\begin{abstract}
To significantly increase the sampling rate of an A/D converter (ADC), a time interleaved ADC system is a good option. The drawback of a time interleaved ADC system is that the ADCs are not exactly identical due to errors in the manufacturing process. This means that time, gain and offset mismatch errors are introduced in the ADC system. These errors cause distortion in the sampled signal.

In this paper we present a method for estimation and compensation of the time mismatch errors. The estimation method requires no knowledge about the input signal except that it should be band limited to the foldover frequency, $\pi / T_{s}$, for the complete ADC system. This means that the errors can be estimated while the ADC is running. The method is also adaptive to slow changes in the time errors.

The Cramer-Rao bound for the time error estimates is also calculated and compared to Monte-Carlo simulations. The estimation method has also been validated on measurements from a real time interleaved ADC system with 16 ADCs.
\end{abstract}

Index Terms-A/D conversion, nonuniform sampling, equalization, estimation

\section{INTRODUCTION}

$\mathbf{T}$ HERE is an ever increasing need for faster A/D converters (ADCs) in modern communications technology, such as radio base stations and VDSL modems. To achieve high enough sample rates, an array of $M$ ADCs, interleaved in time, can be used [1], [2], see Figure 1. The time interleaved ADC system works as follows:

- The input signal is connected to all the ADCs.

- Each ADC works with a sampling interval of $M T_{s}$, where $M$ is the number of ADCs in the array and $T_{s}$ is the desired sampling interval.

- The clock signal to the $i$ th ADC is delayed with $i T_{s}$. This gives an overall sampling interval of $T_{s}$.

The drawback with the interleaved structure is that, due to the manufacturing process, all the ADCs are not identical

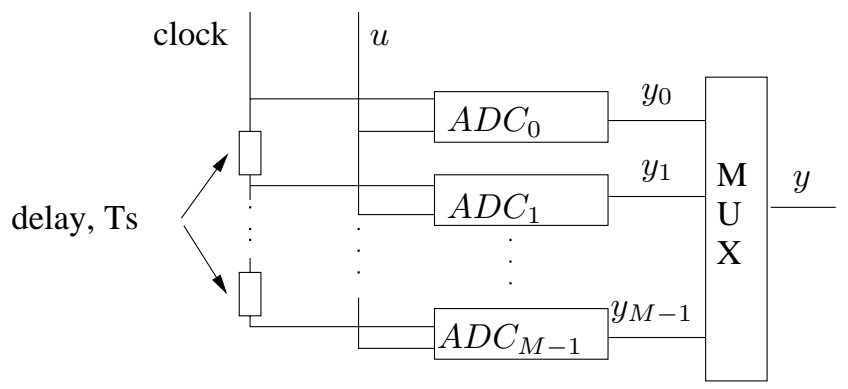

Fig. 1. A time interleaved ADC system. $M$ parallel ADCs are used with the same master clock. The clock is delayed by the nominal sampling interval to each ADC. The outputs are then multiplexed together to form a signal sampled $M$ times faster than the output from each ADC. and mismatch errors will occur in the system. Three kinds of mismatch errors will occur:

\section{- Time errors (static jitter)}

The delay times of the clock between the different ADCs are not equal. This means that the signal will be periodically but non-uniformly sampled.

\section{- Amplitude offset errors}

The ground level differs between the different ADCs. This means that there is a constant amplitude offset in each ADC.

- Gain errors

The gain, from analog input to digital output, differs between the different ADCs.

The errors listed above are static or slowly time varying. This means here that the errors can be assumed to be constant for the same ADC from one cycle to the next over an interval of some million samples.

With a sinusoidal input, the mismatch errors can be seen in the output spectrum as non harmonic distortion [3]. With input signal frequency $\omega_{0}$, the gain and time errors cause distortion at the frequencies

$$
\frac{i}{M} \omega_{s} \pm \omega_{0}, i=1, \ldots, M-1
$$

where $\omega_{s}$ is the sampling frequency. The offset errors cause distortion at the frequencies

$$
\frac{i}{M} \omega_{s}, i=1, \ldots, M-1
$$

An example of an output spectrum from an interleaved ADC system with four ADCs with sinusoidal input signal is shown in Figure 2. This distortion causes problems for instance in a radio receiver where a weak carrier cannot be distinguished from the mismatch distortion from a strong carrier. It is therefore important to remove the mismatch errors. However, calibration of an ADC system is time consuming and costly. Furthermore the mismatch errors may change slowly with for instance temperature and aging. Therefore we want to estimate the mismatch errors while the ADC is used. Methods for estimation of timing errors have been published in for instance [4] and [5]. These methods require a known calibration signal, which means that the operation of the ADC must be stopped during calibration. A blind time error estimation method was presented in [6] and validated on measurements in [7]. This method works well, but gives a bias error in the time error estimates. A blind amplitude offset error estimation method was presented in [8].

We will in this paper present a method for blind equalization of the time mismatch errors in a time interleaved ADC system. 


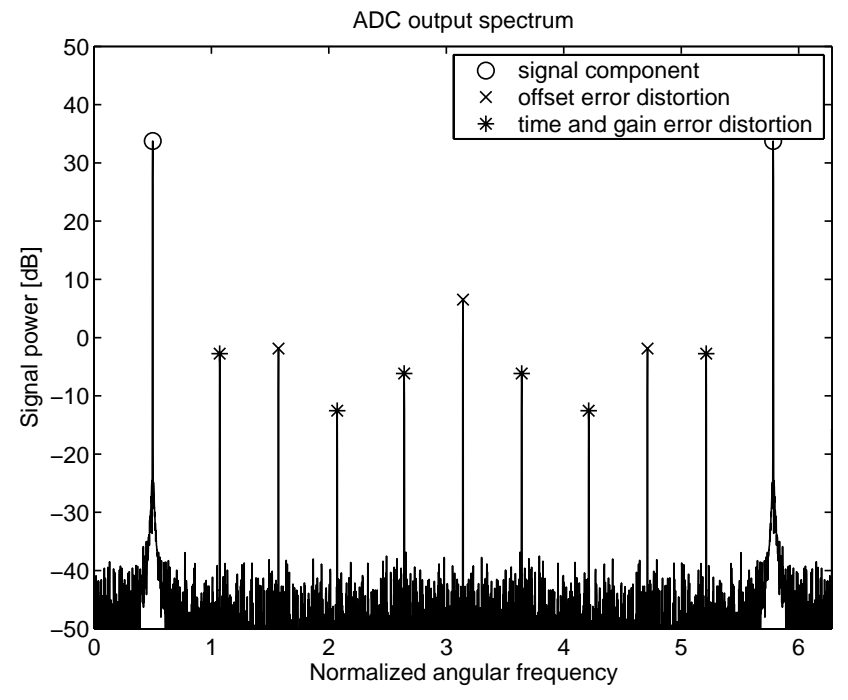

Fig. 2. Simulated output spectrum from interleaved ADC system with four ADCs. The input signal is a single sinusoid. The distortion is caused by mismatch errors.

The estimation method requires only that the input signal is band limited to the foldover frequency, $\pi / T_{s}$, for the complete ADC system. This method gives no bias in the estimates. The joint estimation of all three mismatch error types is presented in [9] where the time error estimation presented in this paper is one part, studied from a system perspective. In this paper, the time error estimation method is studied in more detail.

\section{NOTATION AND DEFINITIONS}

We will in this section introduce the notation that will be used in this paper. The nominal sampling interval, that we would have without time errors, is denoted $T_{s} . M$ denotes the number of ADCs in the time interleaved array, which means that the sampling interval for each $\mathrm{ADC}$ is $M T_{s}$. The time error parameters are denoted $\Delta_{t_{i}}, i=0, \ldots, M-1$. The estimates of these errors are denoted $\hat{\Delta}_{t_{i}}$, and the true time errors are denoted $\Delta_{t_{i}}^{0}$. The vector notation $\Delta_{t}=\left[\Delta_{t_{0}} \cdots \Delta_{t_{M-1}}\right]$ is used for all the time error parameters.

We use the following notation for the signals involved:

- $u(t)$ is the analog input signal.

- $u[k]$ denotes the ideal signal, sampled without mismatch errors.

- $u_{i}[k], i=0, \ldots, M-1$ denotes the $M$ subsequences of $u[k]$,

$$
u_{i}[k]=u[k M+i] .
$$

- $y_{i}[k] i=0, \ldots, M-1$ denotes the output subsequences from the $M \mathrm{~A} / \mathrm{D}$ converters, sampled with time errors.

$$
y_{i}[k]=u\left((k M+i) T_{s}+\Delta_{t_{i}}^{0}\right)
$$

- $y[k]$ is the multiplexed output signal from all the ADCs,

$$
y[k]=y_{(k \bmod M)}\left[\left\lfloor\frac{k}{M}\right\rfloor\right],
$$

where $\lfloor\cdot\rfloor$ denotes rounding towards $-\infty$.
- $z^{\left(\Delta_{t}\right)}[k]$ denote the output signal, $y[k]$, reconstructed with the error parameters, $\Delta_{t}$.

- $z_{i}^{\left(\Delta_{t}\right)}[k]$ are the subsequences of $z^{\left(\Delta_{t}\right)}[k]$

We assume throughout this paper that $u(t)$ is band limited to the foldover frequency, $\frac{\pi}{T_{s}}$, of the complete ADC system.

We will next establish a few definitions which will be used later in the paper. A discrete time signal $u[k]$ is said to be quasi-stationary [10] if

$$
\begin{aligned}
& \bar{m}_{u}=\lim _{N \rightarrow \infty} \frac{1}{N} \sum_{k=1}^{N} E(u[k]) \\
& \bar{R}_{u}[n]=\lim _{N \rightarrow \infty} \frac{1}{N} \sum_{n=1}^{N} E(u[k+n] u[k])
\end{aligned}
$$

both exist, where the expectation is taken over possible stochastic parts of the signal. Analogously, a continuous time signal $u(t)$ is quasi-stationary if

$$
\begin{aligned}
& \bar{m}_{u}=\lim _{T \rightarrow \infty} \frac{1}{T} \int_{0}^{T} E(u(t)) d t \\
& \bar{R}_{u}(\tau)=\lim _{T \rightarrow \infty} \frac{1}{T} \int_{0}^{T} E(u(t+\tau) u(t)) d t
\end{aligned}
$$

both exist. A stationary stochastic process is quasi-stationary, with $\bar{m}_{u}$ and $\bar{R}_{u}[n]$ being the mean value and covariance function respectively.

\section{Definition 1 (Modulo M quasi-stationary) Assume}

$$
\begin{aligned}
& \bar{g}_{u_{i_{1}}, u_{i_{2}}, \ldots}=\lim _{N \rightarrow \infty} \frac{1}{N} \sum_{t=1}^{N} g\left(u_{i_{1}}[t], u_{i_{2}}[t], \ldots\right), \\
& i_{1}, i_{2}, \cdots=0, \ldots, M-1
\end{aligned}
$$

exists for a function $g(\cdot, \cdot, \cdots)$. Then $u$ is modulo $M$ quasistationary with respect to $g$ if

$$
\begin{aligned}
& \bar{g}_{i_{1}, i_{2}, \cdots}=\bar{g}_{\left\{\left(i_{1}+l\right) \bmod M,\left(i_{2}+l\right) \bmod M, \cdots\right\}}, \\
& \forall l \in\{\ldots,-1,0,1, \ldots\}
\end{aligned}
$$

The modulo $M$ quasi-stationarity property guarantees that the input signal has the same statistical properties for all the ADCs in the time interleaved system. We assume throughout the rest of the paper that the input signal is modulo $M$ quasi-stationary with respect to $g_{1}\left(u_{i}\right)=u_{i}, g_{2}\left(u_{i}\right)=u_{i}^{2}$ and $g_{3}\left(u_{i}, u_{j}\right)=$ $\left(u_{i}-u_{j}\right)^{2}$.

\section{Example 1 (Modulo $M$ quasi-stationary) Consider first the function}

$$
g\left(u_{i}[k]\right)=u_{i}^{2}[k] .
$$

The modulo $M$ quasi-stationary property then means that the mean square value should be equal for all subsequences, i.e., if

$$
\bar{\sigma}_{i}^{2}=\lim _{N \rightarrow \infty} \frac{1}{N} \sum_{k=1}^{N} u_{i}^{2}[k]
$$

then

$$
\bar{\sigma}_{i}^{2}=\bar{\sigma}_{j}^{2}, i, j=0, \ldots, M-1 .
$$


In this example this is true for most quasi-stationary signals, but some periodic signals are not modulo $M$ quasi-stationary. Consider the deterministic signal

$$
u[k]=\cos \left(\frac{\pi}{2} k\right)
$$

and $M=2$. Then we have

$$
\bar{\sigma}^{2}=\lim _{N \rightarrow \infty} \frac{1}{N} \sum_{k=1}^{N} \cos ^{2}\left(1 \frac{\pi}{2} k\right)=\frac{1}{2}
$$

so the signal is quasi-stationary, but

$$
\bar{\sigma}_{0}^{2}=\lim _{N \rightarrow \infty} \frac{1}{N} \sum_{k=1}^{N} \cos ^{2}\left(2 \frac{\pi}{2} k\right)=1
$$

and

$$
\bar{\sigma}_{1}^{2}=\lim _{N \rightarrow \infty} \frac{1}{N} \sum_{k=1}^{N} \cos \left(2 \frac{\pi}{2} k+\frac{\pi}{2}\right)=0
$$

i.e., the signal is not modulo 2 quasi-stationary with respect to $g\left(u_{i}[k]\right)=u_{i}^{2}[k]$, but it is with respect to $g\left(u_{i}[k]\right)=u_{i}[k]$.

\section{Signal Reconstruction With KNOWn Time ERRORS}

If the time error parameters are known, and the input signal $u(t)$ is band limited to the Nyquist frequency, $u(t)$ can be exactly reconstructed from the sampled signal $y[k]$. We will in this section describe the signal reconstruction.

The time errors can be compensated for by many different interpolation techniques, for instance splines [13], polynomial interpolation or filter bank interpolation [14]. We will here describe a method for exact interpolation by filtering the signal with a non-causal IIR filter. If the input signal is band limited to the foldover frequency, $\frac{\pi}{T_{s}}$, and the time error parameters are known, the input signal can be perfectly reconstructed from the irregular samples [15]. In a real application, the interpolation is of course approximate since we cannot use a filter of infinite length, but we can come arbitrarily close to the exact interpolation by choosing the length of the filter large enough. In [15] the interpolation is done at an arbitrary time instance according to the following:

Solve the equation system

$$
\begin{aligned}
& \sum_{i=0}^{M-1} e^{j\left(-\frac{M-1}{2}+i+\Delta_{t_{i}}\right) \omega} H_{i}(\omega, t)=1 \\
& \sum_{i=0}^{M-1} e^{j\left(-\frac{M-1}{2}+i+\Delta_{t_{i}}\right)\left(\omega+\frac{2 \pi}{M T_{S}}\right)} H_{i}(\omega, t)=e^{j \frac{2 \pi}{M T_{S}} t} \\
& \sum_{i=0}^{M-1} e^{j\left(-\frac{M-1}{2}+i+\Delta_{t_{i}}\right)\left(\omega+(M-1) \frac{2 \pi}{M T_{s}}\right)} H_{i}(\omega, t)=e^{j(M-1) \frac{2 \pi}{M T_{s}} t}
\end{aligned}
$$

for $H_{i}(\omega, t)$. The input signal can then be calculated at any time instance as

$$
u(t)=\sum_{k=-\infty}^{\infty} \sum_{i=0}^{M-1} y_{i}[k] h_{i}\left(t-k M T_{s}\right)
$$

where

$$
h_{i}(t)=\frac{M T_{s}}{2 \pi} \int_{-\pi / T_{s}}^{-\pi / T_{s}+2 \pi /\left(M T_{s}\right)} H_{i}(\omega, t) e^{j \omega t} d \omega
$$

The reconstruction described in [15] is done at an arbitrary time instance and is quite computationally demanding. If we only need to reconstruct the signal at the nominal sampling instances

$$
t=(k M+l) T_{s}, l=0, \ldots, M-1, k=\ldots,-1,0,1, \ldots
$$

the reconstruction can be computationally simplified. Here we introduce the notation $\alpha_{i}=-\frac{M-1}{2}+i+\Delta_{t_{i}}$, to simplify the equation system (2). The right hand side of (2) is then independent of $k$ in (3) and depends only on $l$. Further, the left hand side can be factorized into one diagonal matrix which depends on $\omega$, one matrix independent of $\omega$ and $H(\omega, t)$ which now also is independent of $k$

$$
A(\alpha) E(\alpha, \omega) H^{(l)}(\omega)=B_{l}
$$

Here

$$
\begin{gathered}
A(\alpha)=\left[\begin{array}{ccc}
1 & \cdots & 1 \\
e^{j \alpha_{0} \frac{2 \pi}{M T_{S}}} & \cdots & e^{j \alpha_{M-1} \frac{2 \pi}{M T_{s}}} \\
\vdots & \ddots & \vdots \\
e^{j \alpha_{0}(M-1) \frac{2 \pi}{M T_{s}}} & \cdots & e^{j \alpha_{M-1}(M-1) \frac{2 \pi}{M T_{s}}}
\end{array}\right] \\
E(\alpha, \omega)=\left[\begin{array}{cccc}
e^{j \alpha_{0} \omega} & 0 & \cdots & 0 \\
0 & e^{j \alpha_{1} \omega} & \cdots & 0 \\
\vdots & \vdots & \ddots & \vdots \\
0 & 0 & \cdots & e^{j \alpha_{M-1} \omega}
\end{array}\right]
\end{gathered}
$$

and

$$
B_{l}=\left[\begin{array}{llll}
1 & e^{j 2 \pi l / M} & \cdots & e^{j 2 \pi(M-1) l / M}
\end{array}\right]^{T}
$$

Since only $E(\alpha, \omega)$ depends on $\omega$ and the time dependence in the right hand side of (2) is removed, we can easily calculate the coefficients $h_{i}^{(l)}[k]=h_{i}\left((k M+l) T_{s}\right)$

$$
\begin{aligned}
& h^{(l)}[k]= \\
& \frac{M T_{s}}{2 \pi} \int_{-\pi / T_{s}}^{-\pi / T_{s}+2 \pi /\left(M T_{s}\right)} E^{-1}(\alpha, \omega) e^{j \omega(k M+l) T_{s}} d \omega A^{-1}(\alpha) B_{l}
\end{aligned}
$$

From here on we assume $M$ to be even, $M$ odd gives similar calculations. Calculating the DTFT (discrete-time Fourier 
transform) of the subsequences $h^{(l)}[k]$ gives

$$
\begin{aligned}
& H^{(l)}\left(e^{j \omega M T_{s}}\right)=M T_{s} \sum_{k=-\infty}^{\infty} h^{(l)}[k] \\
& =\frac{\left(M T_{s}\right)^{2}}{2 \pi} \int_{-\pi / T_{s}}^{-\pi / T_{s}+2 \pi /\left(M T_{s}\right)} E^{-1}(\alpha, \gamma) e^{j \gamma l T_{s}} \\
& \sum_{k=-\infty}^{\infty} e^{j \gamma k M T_{s}} e^{-j \omega k M T_{s}} d \gamma A^{-1}(\alpha) B_{l} \\
& =M T_{s} \sum_{r=-\infty}^{\infty} \int_{-\pi / T_{s}}^{-\pi / T_{s}+2 \pi /\left(M T_{s}\right)} E^{-1}(\alpha, \gamma) e^{j \gamma l T_{s}} \\
& \delta\left(\gamma-\omega+r \frac{2 \pi}{M T_{s}}\right) d \gamma A^{-1}(\alpha) B_{l} \\
& =M T_{s} E^{-1}\left(\alpha, \omega-\frac{\pi}{T_{s}}\right) e^{j \omega l T_{s}}(-1)^{l} A^{-1}(\alpha) B_{l}, \\
& 0 \leq \omega<\frac{2 \pi}{M T_{s}}
\end{aligned}
$$

The subsequences $Z_{i}^{\left(\Delta_{t}\right)}\left(e^{j \omega M T_{s}}\right)$ can then be calculated as

$$
\begin{aligned}
& Z_{l}^{\left(\Delta_{t}\right)}\left(e^{j \omega M T_{s}}\right) \\
& =Y^{T}\left(e^{j \omega M T_{s}}\right) M T_{s} E^{-1}\left(\alpha, \omega-\frac{\pi}{T_{s}}\right) e^{j \omega l}(-1)^{l} A^{-1}(\alpha) B_{l}
\end{aligned}
$$

where

$$
Y^{T}\left(e^{j \omega M T_{s}}\right)=\left[\begin{array}{lll}
Y_{0}\left(e^{j \omega M T_{s}}\right) & \cdots & Y_{M-1}\left(e^{j \omega M T_{s}}\right)
\end{array}\right]
$$

The DTFT of the time error compensated signal, $Z^{\left(\Delta_{t}\right)}\left(e^{j \omega T_{s}}\right)$, can then be calculated from its subsequences [17]

$$
Z^{\left(\Delta_{t}\right)}\left(e^{j \omega T_{s}}\right)=\sum_{l=0}^{M-1} Z_{l}^{\left(\Delta_{t}\right)}\left(e^{j\left(\omega M T_{s} \bmod 2 \pi\right)}\right) e^{-j l \omega T_{s}}
$$

With the inverse DTFT we get the time error reconstructed signal

$$
z^{\left(\Delta_{t}\right)}[k]=D T F T^{-1}\left(Z^{\left(\Delta_{t}\right)}\left(e^{j \omega T_{s}}\right)\right)
$$

In practice (7), (8) and (9) are calculated on finite sequences using the DFT (discrete Fourier transform) instead of the DTFT.

\section{TIME ERROR ESTIMATION}

We will in this section present a method to estimate the time errors in a time interleaved ADC system. The estimation is done without a special calibration signal and without knowledge of the input signal.

Now, since the input signal $u[k]$ is modulo $M$ quasistationary and thus does not contain any deterministic periodic components in phase with the sampling frequency, the output signal $y[k]$ is $M$-cyclostationary [11], [12]. Also the reconstructed signal $z[k]$ (9) will then be $M$-cyclostationary for all time error estimates $\Delta_{t}$. We can then define the vector process $\mathbf{z}^{\left(\Delta_{\mathbf{t}}\right)}[k]$ as

$$
\mathbf{z}^{\left(\Delta_{\mathbf{t}}\right)}[k]=\left[\begin{array}{llll}
z_{0}^{\left(\Delta_{t}\right)}[k] & z_{1}^{\left(\Delta_{t}\right)}[k] & \cdots & z_{M-1}^{\left(\Delta_{t}\right)}[k]
\end{array}\right]^{T}
$$

and its corresponding $M \times M$ covariance function

$$
\mathbf{R}_{\mathbf{z}}^{\Delta_{\mathbf{t}}}[l]=\lim _{N \rightarrow \infty} \frac{1}{N} \sum_{k=1}^{N}\left\{\mathbf{z}^{\left(\Delta_{\mathbf{t}}\right)}[k]\left(\mathbf{z}^{\left(\Delta_{\mathbf{t}}\right)}\right)^{T}[k+l]\right\} .
$$

Note that M-cyclostationarity implies that (11) exists for all $\Delta_{t}$. Note also that $\mathbf{z}^{\left(\Delta_{\mathbf{t}}\right)}$ becomes quasi-stationary when the true time errors $\hat{\Delta}_{t}=\Delta_{t}^{0}$ are found. In that case, its covariance function $\mathbf{R}_{\mathbf{z}}^{\Delta_{\mathbf{t}}}[l]$ becomes Toeplitz for each value of $l$. Basically, that is the only second order information we have in the blind equalization case. Thus, it seems natural to consider norms of how far the covariance function is from Toeplitz, and minimize this norm with respect to $\Delta_{t}$. A quite general norm that measures the quadratic difference on each diagonal of $\mathbf{R}_{\mathbf{z}}^{\Delta_{t}}$ is given by

$$
V\left(\Delta_{t}\right)=\sum_{l=0}^{\infty} \sum_{m=0}^{M-1} \sum_{i, j}\left(\left(R_{z}^{\Delta_{t}}\right)_{i, i-m}[l]-\left(R_{z}^{\Delta_{t}}\right)_{j, j-m}[l]\right)^{2}
$$

where $\left(R_{z}^{\Delta_{t}}\right)_{i, j}[l]$ is the element on row $i$ and column $j$ in $\mathbf{R}_{\mathbf{z}}^{\Delta_{\mathbf{t}}}[l]$.

Clearly, for a correlated signal $\mathrm{u}$, the most information is found for small lags in its covariance function, which for the vector process $\mathbf{z}$ corresponds to the first diagonal in the off-diagonal part of the covariance function. We will in the following consider the special case of (12) where only the first off-diagonal diagonal is studied for zero lag $(l=0)$ :

$$
V_{1}\left(\Delta_{t}\right)=\sum_{i, j}\left(\left(R_{z}^{\Delta_{t}}\right)_{i, i-1}[0]-\left(R_{z}^{\Delta_{t}}\right)_{j, j-1}[0]\right)^{2} .
$$

We will in the following assume that the time error in the first ADC is zero, i.e., $\Delta_{t_{0}}^{0}=0$. This is no loss of generality since only the distance between the samples, and not the absolute sampling instances, needs to be correct.

The signal reconstruction described in Section III is quite complicated to analyze. Also other signal reconstruction approximations can be used. Therefore we first study the loss function (13) assuming a signal reconstruction where the time error parameters change the time errors linearly. This means that we can study the output signals

$$
\begin{aligned}
& y_{0}[k]=u\left(M k T_{s}\right) \\
& y_{i}[k]=u\left((M k+i) T_{s}+\Delta_{t_{i}}\right), i=1, \ldots, M-1 .
\end{aligned}
$$

parameterized in the time error parameters, $\Delta_{t_{i}}$. In the next section we will discuss how the reconstruction described in Section III affects the time error estimation.

Next, we will state a few theroems about the loss function (13). The proofs for these theorems are given in [16].

First we consider a dual ADC system, i.e., $M=2$. The first theorem says that we have a global minimum for the correct time error parameters. The second theorem says that the loss function is monotonically increasing around this minimum.

Theorem 1 Consider the loss function (13) and assume that reconstruction with the time error parameters changes the time linearly, i.e., the model (14) is used. Assume then that $\left|\Delta_{t}\right|<$ 
$T_{s}$ and $T_{s}<\pi / \omega_{c}$, where $\omega_{c}$ is the bandwidth of the input signal. Then

$$
V_{1}(0)=0
$$

and

$$
V_{1}\left(\Delta_{t}\right)>0, \text { if } \Delta_{t} \neq 0
$$

Theorem 2 Consider the assumptions given in Theorem 1. Assume that $\left|\Delta_{t}\right|<T_{s} / 2$ and $T_{s}<\pi / \omega_{c}$, where $\omega_{c}$ is the bandwidth of the input signal. Then $V_{1}^{\infty}\left(\Delta_{t}\right)$ is monotonically increasing around $\Delta_{t}=0$.

This means that as long as we have a Nyquist sampling system and the time errors are smaller than half the sampling interval, we can estimate the time errors.

For the general case $(M \geq 2)$ we cannot guarantee a global minimum for $V_{1}\left(\Delta_{t}\right)$ for any signal arbitrarily close to the foldover frequency. However, we do not have to change the requirements much.

Theorem 3 Consider the assumptions given in Theorem 1. Assume that $\left|\Delta_{t}\right|<T_{s} / 2$ and $T_{s}<\frac{4}{5} \frac{\pi}{\omega_{c}}$. Then $V_{1}(0)=0$ and $V_{1}\left(\Delta_{t}\right)>0$ if $\Delta_{t} \neq 0$.

If we know that the time error parameters are smaller we can allow an input signal closer to the foldover frequency and when $\max \left|\Delta_{t_{i}}\right| \rightarrow 0$ we only need the requirement that $T_{s}<$ $\pi / \omega_{c}$.

\section{Estimation ALgOrithm}

In this section we will discuss how the time errors can be estimated practically using the theory from the previous sections, but with some modifications to incorporate the actual signal reconstruction used.

With the reconstruction described in Section III, simulations show that there are local minima in the loss function $V_{1}\left(\Delta_{t}\right)$. A contour plot of $V_{1}\left(\Delta_{t}\right)$ is shown in Figure 3. Here $M=4$, and $\Delta_{t_{0}}$ and $\Delta_{t_{2}}$ are fixed to their true values to generate a two-dimensional plot. The input signal is here sinusoidal. We can see that there are local minima along a line, $\Delta_{t_{1}}-$ $\Delta_{t_{3}}=$ constant, in this figure. However, when $\Delta_{t} \neq \Delta_{t}^{0}$ in the interpolation, simulations show that the gain of the subsequences of the interpolated signals are changed, i.e., the main diagonal in the covariance function $\mathbf{R}_{\mathbf{z}}^{\Delta_{\mathbf{t}}}[l]$ is no longer constant. Consider instead the part of the loss function (12) that involves the main diagonal

$$
V_{0}\left(\Delta_{t}\right)=\sum_{i, j}\left(\left(R_{z}^{\Delta_{t}}\right)_{i, i}[l]-\left(R_{z}^{\Delta_{t}}\right)_{j, j}[l]\right)^{2} .
$$

If we plot the same contour plot for this function, see Figure 4, we see that again there are local minima along a line. But this line, $\Delta_{t_{1}}+\Delta_{t_{3}}=$ constant, is perpendicular to the line in Figure 3. This means that adding the two loss functions (13) and (15) we still get a special case of (12):

$$
V_{0,1}\left(\Delta_{t}\right)=V_{0}\left(\Delta_{t}\right)+V_{1}\left(\Delta_{t}\right)
$$

Simulations indicate that this loss function eliminates the local minima, see Figure 5. This is just an example with a sinusoidal input, but simulations of many different input signals with different frequency range and different values of $M$ indicate that this loss function works for a wide range of signals.

The minimizing arguments of the loss function (16) gives the time error estimates. Since the minimizing argument cannot be calculated analytically, a numerical minimization algorithm is used. Further, the mismatch errors may change slowly with for instance temperature and aging. Therefore the parameter estimates should be adaptively updated with new data. There are many minimization algorithms available with fast convergence, for instance Newton's method [18]. However, the fast converging methods are usually computationally demanding. Therefore a stochastic gradient search method is chosen here, which has a somewhat slower convergence rate than other methods, but is computationally very efficient. In a stochastic gradient minimization algorithm, the parameters are updated by a step in the negative gradient direction Here,

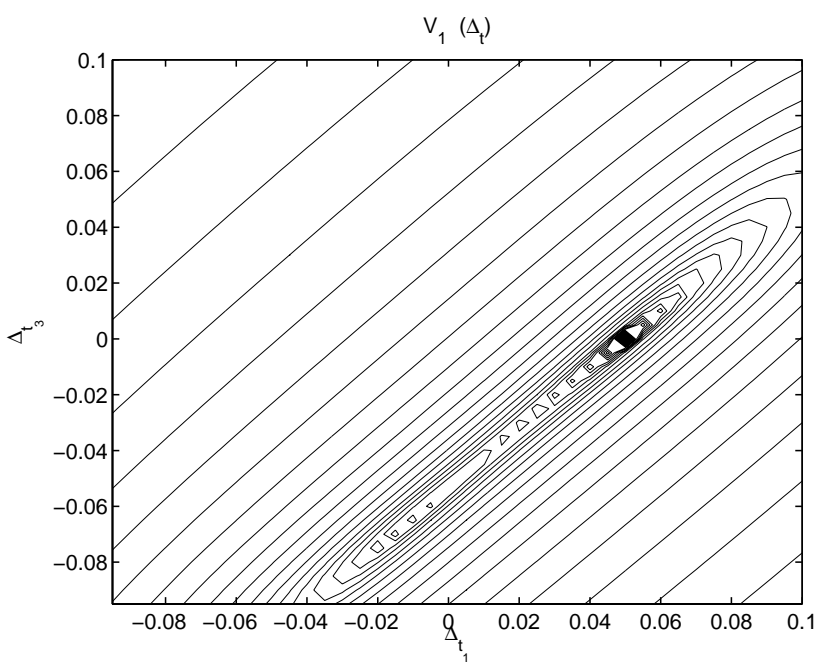

Fig. 3. A contour plot of the time error loss function, $V_{1}\left(\Delta_{t}\right)$, with $M=4$ and sinusoidal input. $\Delta_{t_{0}}$ and $\Delta_{t_{2}}$ are fixed to their true values.

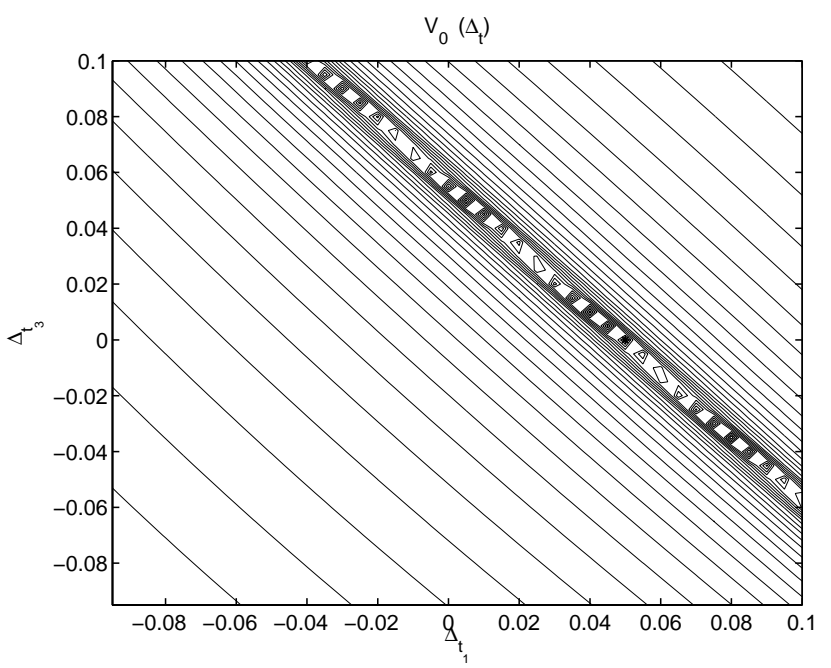

Fig. 4. A contour plot of the time error loss function, $V_{0}\left(\Delta_{t}\right)$, with $M=4$ and sinusoidal input. $\Delta_{t_{0}}$ and $\Delta_{t_{2}}$ are fixed to their true values. 
a normalized version of the stochastic gradient method is used to make the choice of $\mu$ easier

$$
\hat{\Delta}_{t}^{(i+1)}=\hat{\Delta}_{t}^{(i)}-\mu \frac{\nabla V\left(\hat{\Delta}_{t}^{(i)}\right)}{\max \left|\nabla V\left(\hat{\Delta}_{t}^{(i)}\right)\right|}
$$

To avoid taking too long steps, we can check that the loss function decreases for every iteration, and otherwise backtrack the step size until it does [18]. The next iteration is then started with doubled step length, so that the step length does not get unnecessarily small. To summarize, the adaptive equalization algorithm is given by

\section{Algorithm 1 (Interleaved ADC equalization) Initialization:}

- Choose a batch size, $N$, for each iteration.

- Initialize the step lengths of the stochastic gradient algorithm, $\mu_{t}$. If the order of magnitude of the mismatch errors are known, this information can be used for the initialization.

- Initialize the parameter estimates

$$
\hat{\Delta}_{t_{i}}^{(0)}=0, i=0, \ldots, M-1
$$

\section{Adaptation:}

1) Collect a batch of $N$ data from each $A D C, y_{i}[k], i=$ $0, \ldots, M-1$.

2) Calculate the reconstructed signals

$$
z_{i}^{\left(\hat{\Delta}_{t}^{(j)}\right)}[k], i=0, \ldots, M-1
$$

according to (7), (8) and (9)

3) Calculate the gradient of the loss function, $\nabla V_{0,1}\left(\hat{\Delta}_{t}^{(j)}\right)$. The gradients can be calculated numerically by a finite difference approximation from the loss functions, or by analytically differentiating the loss function. The loss function is defined in (16).

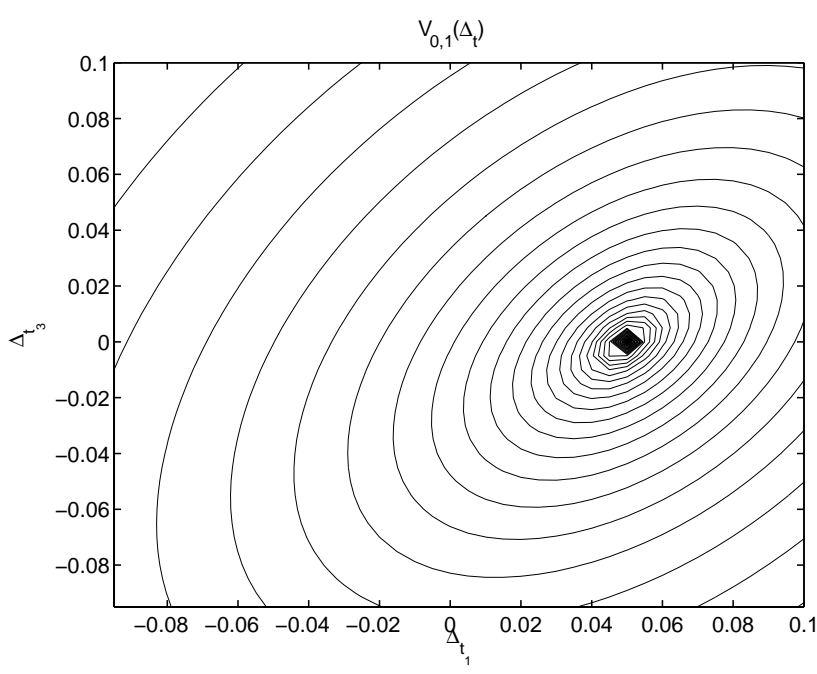

Fig. 5. A contour plot of the time error loss function, $V_{0,1}\left(\Delta_{t}\right)=V_{0}\left(\Delta_{t}\right)+$ $V_{1}\left(\Delta_{t}\right)$, with $M=4$ and sinusoidal input. $\Delta_{t_{0}}$ and $\Delta_{t_{2}}$ are fixed to their true values.
4) Update the parameter estimates

$$
\hat{\Delta}_{t}^{(j+1)}=\hat{\Delta}_{t}^{(j)}-\mu_{t} \frac{\nabla V_{0,1}\left(\hat{\Delta}_{t}^{(j)}\right)}{\max \left|\nabla V_{0,1}\left(\hat{\Delta}_{t}^{(j)}\right)\right|}
$$

5) If the loss function has increased since the last iteration

$$
V_{0,1}\left(\hat{\Delta}_{t}^{(j+1)}\right)>V_{0,1}\left(\hat{\Delta}_{t}^{(j)}\right)
$$

backtrack the step size $\mu_{t}:=\mu_{t} / 2$ and change the parameter estimates in step 4) until the loss function decreases. Otherwise double the step lengths for the next iteration: $\mu_{t}:=2 \mu_{t}$.

6) Return to step 1).

Figure 6 illustrates the operation of the adaptive equalization algorithm.

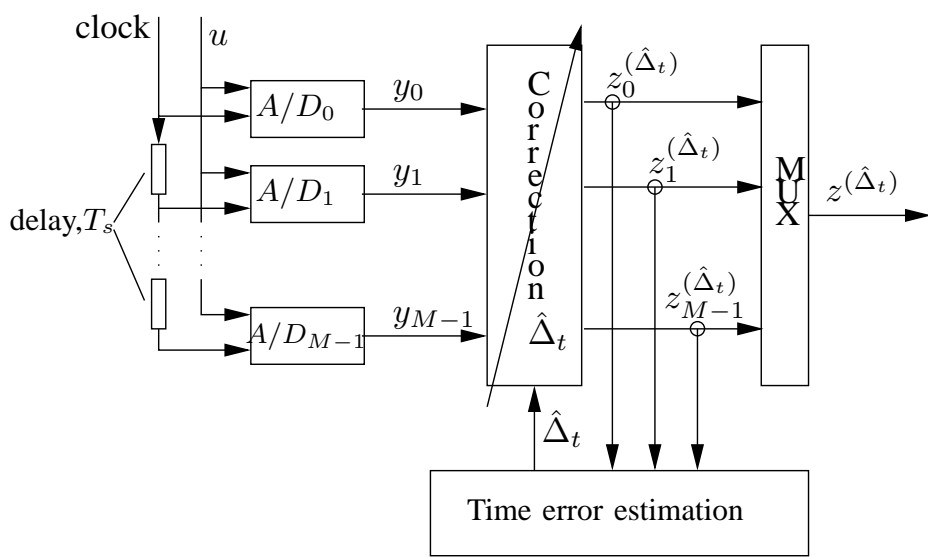

Fig. 6. Time interleaved ADC system with time errors. The time errors, $\hat{\Delta}_{t}$, are estimated by a blind adaptive algorithm and the signal is corrected by a filter.

The signal correction part of the algorithm must be done in real time. However, the estimation part may work more slowly depending on how fast the time errors change. The signal correction is done on a batch of data containing $N$ samples per ADC. The correction involves a DFT and inverse DFT calculation which requires $N \log _{2} N$ operations each per ADC. The matrices $A(\alpha), E(\alpha, \omega)$ and $B_{l}$ in (4-6) do not have to be updated for each batch of data, assuming that the estimation is done more slowly. This means that these matrices can be precalculated and the calculation of the corrected signal therefore requires $M N$ operations per ADC. This gives a total of $M^{2} N+2 M N \log _{2} N$ operations for the correction for each batch of data, i.e., $M+2 \log _{2} N$ operations per sample. One operation is here basically one multiplication and one summation.

The estimation part requires more operations, but these calculations can normally be done at a slower rate and are therefore not critical.

This algorithm is intended for applications that require high sample rates, such as soft radio base stations and VDSL modems. This means that a lot of data is available and the algorithm will therefore converge in a fraction of a second. In these systems there are also a lot of computational power available on chip which means that is should be possible to implement this algorithm in existing hardware on chip. 


\section{CRAMER-RAO BOUND}

The Cramer-Rao bound (CRB) is a lower bound on the variance of the estimated parameters for a given amount of data [10], independent of the estimation algorithm used. The CRB is here calculated assuming known input while the estimation algorithm is blind. This means that the estimation algorithm never can reach the CRB. The reason for this is that the blind $\mathrm{CRB}$ is difficult to calculate. However, the non-blind CRB still gives a rule of thumbs of the estimation performance and it is simple to interpret in terms of design parameters and input signals. We will first calculate the CRB for a general input signal assuming only additive noise on the signal. The CRB is here calculated asymptotically for a general input signal. For a special signal it is also possible to calculate the $\mathrm{CRB}$ for a fixed, finite amount of data, but to keep it general this is not included here. With stochastic jitter we cannot calculate a general expression for the CRB, but we will calculate the $\mathrm{CRB}$ for some special input signals.

\section{A. CRB for additive noise}

Assuming only additive noise, the output signal subsequences are

$$
\begin{aligned}
& y_{0}[k]=u\left(M k T_{s}\right)+e_{0}[k] \\
& y_{i}[k]=u\left((M k+i) T_{s}+\Delta_{t_{i}}^{0}\right)+e_{i}[k], i=1, \ldots, M-1
\end{aligned}
$$

The noise is here assumed to be white Gaussian

$$
e_{i}[k] \in N\left(0, \sigma_{e}\right)
$$

despite that uniformly distributed noise is a better model of the quantization noise. However, Gaussian noise simplifies the calculations of the CRB and simulations show that, with the same noise variance, the estimation accuracy is approximately the same for Gaussian and uniform noise. The parameterized signal model is

$$
\begin{aligned}
& \hat{y}_{0}[k]=u\left(M k T_{s}\right) \\
& \hat{y}_{i}[k]=u\left((M k+i) T_{s}+\Delta_{t_{i}}\right), \quad i=1, \ldots, M-1
\end{aligned}
$$

The negative log-likelihood function [10] is then calculated by taking the logarithm of the probability density function of the noise.

$$
\begin{aligned}
& -\lim _{N \rightarrow \infty} \log f_{e}\left(\Delta_{t}, y^{N}\right) \\
& =\lim _{N \rightarrow \infty} \frac{1}{N} \frac{1}{2 \sigma_{e}^{2} M} \sum_{i=0}^{M-1} \sum_{k=1}^{N}\left\{y_{i}[k]-\hat{y}_{i}[k]\right\}^{2} \\
& =\frac{1}{2 \sigma_{e}^{2} M} \sum_{i=0}^{M-1}\left\{\left(2 \sigma_{u}^{2}+\sigma_{e}^{2}\right)-2 R_{u}\left(\Delta_{t_{i}}^{0}-\Delta_{t_{i}}\right)\right\}
\end{aligned}
$$

Differentiating the log-likelihood function twice with respect to the error parameters gives

$$
\begin{aligned}
& \frac{d^{2}}{d \Delta^{2}}\left(-\lim _{N \rightarrow \infty} \log f_{e}\left(\Delta_{t}, y^{N}\right)\right)=\frac{1}{2 \sigma_{e}^{2} M} \cdot \\
& {\left[\begin{array}{ccc}
-2 \frac{d^{2} R_{u}\left(\Delta_{t_{1}}^{0}-\Delta_{t_{1}}\right)}{\left(d \Delta_{t_{1}}\right)^{2}} & \cdots & 0 \\
\vdots & \ddots & \vdots \\
0 & \cdots & -2 \frac{d^{2} R_{u}\left(\Delta_{t_{M-1}}^{0}-\Delta_{t_{M-1}}\right)}{\left(d \Delta_{t_{M-1}}\right)^{2}}
\end{array}\right]}
\end{aligned}
$$

Evaluating (17) at $\Delta_{t}=\Delta_{t}^{0}$ gives the Fisher information matrix,

$$
F=-\frac{1}{M \sigma_{e}^{2}} R_{u}^{\prime \prime}(0) I_{(M-1) \times(M-1)}
$$

The Fisher information matrix gives a lower bound on the covariance of the parameter estimates. If the parameters are estimated from $N$ samples per ADC, the Cramer-Rao bound is

$$
\operatorname{Cov}\left(\hat{\Delta}_{t}\right) \geq \frac{1}{M N} F^{-1}
$$

Putting (18) into (19) we get

$$
\operatorname{Var}\left(\hat{\Delta}_{t_{i}}\right) \geq \frac{\sigma_{e}^{2}}{N R_{u}^{\prime \prime}(0)}
$$

We can see from (20) that the CRB for the time error depends on the input signal. We will next evaluate the CRB for a few signal examples.

- Sinusoidal input: In radio applications a single modulated sinusoidal carrier is often used. Here we discard the modulation and calculate the CRB for a sinusoidal signal:

$$
u(t)=\sqrt{2} \sin (\omega t) .
$$

This gives the Cramer-Rao bound

$$
\operatorname{Var}\left(\hat{\Delta}_{t_{i}}\right) \geq \frac{\sigma_{e}^{2}}{N \omega^{2}}
$$

- Multisine input: In DSL modems and OFDM radio communications, a sum of several sinusoidal carriers are used. Here we calculate the CRB for a multisine input signal:

$$
u(t)=\sum_{i=1}^{L} \alpha_{i} \sin \left(\omega_{i} t\right), \frac{1}{2} \sum_{i=1}^{L} \alpha_{i}^{2}=1
$$

This gives the Cramer-Rao bound

$$
\operatorname{Var}\left(\hat{\Delta}_{t_{i}}\right) \geq \frac{2 \sigma_{e}^{2}}{N \sum_{i=1}^{L} \alpha_{i}^{2} \omega_{i}^{2}}
$$

\section{- Band limited white noise input:}

Here the input is a stochastic process with spectrum

$$
\Phi_{u}(\omega)= \begin{cases}\frac{\pi}{\omega_{\max }(1-\alpha)} & \alpha \omega_{\max } \leq|\omega| \leq \omega_{\max } \\ 0 & \text { otherwise }\end{cases}
$$

This gives the Cramer-Rao bound

$$
\operatorname{Var}\left(\hat{\Delta}_{t_{i}}\right) \geq \frac{3 \sigma_{e}^{2}}{N\left(\alpha^{2}+\alpha+1\right) \omega_{\max }^{2}}
$$

The CRB is evaluated in more detail in [16]. 


\section{B. CRB for noise and jitter}

Here we will evaluate the CRB with both noise and stochastic jitter present. The output signal subsequences are now

$$
\begin{aligned}
& y_{0}[k]=u\left(k M T_{s}+e_{0}^{j i t t e r}[k]\right)+e_{0}[k] \\
& y_{i}[k]=u\left((k M+i) T_{s}+\Delta_{t_{i}}^{0}+e_{i}^{j i t t e r}[k]\right)+e_{i}[k], \\
& i=1, \ldots, M-1
\end{aligned}
$$

We assume that both the noise and the random jitter are Gaussian distributed

$$
\begin{aligned}
& e_{i}[k] \in N\left(0, \sigma_{e}\right) \\
& e_{i}^{j i t t e r}[k] \in N\left(0, \sigma_{j i t t e r}\right)
\end{aligned}
$$

Here we cannot, in general, assume that the output signal at a certain time instance is Gaussian distributed. But if we take a sum over many samples we have, according to the central limit theorem [19], that

$$
\bar{y}=\frac{1}{N M} \sum_{k=0}^{N-1} \sum_{i=0}^{M-1} y_{i}[k]
$$

is Gaussian distributed. If we assume that $y[k]$ is modulo $M$ quasistationary with respect to $g\left(u_{i}\right)=u_{i}$ the mean value of $\varepsilon\left(\Delta_{t}, y^{N}\right)$ is zero, independent of the input signal shape. However, the variance depends on what input signal we have. We will therefore in the following consider a few special cases. The derivations are omitted here, but are given in [16].

- Sinusoidal input: $u(t)=\sqrt{2} \sin \left(\omega_{0} t\right)$.

The CRB is here given by

$$
\operatorname{Var}\left(\hat{\Delta}_{t_{i}}\right) \gtrsim \frac{\sigma_{\text {jitter }}^{2}}{N}+\frac{\sigma_{e}^{2}}{N \omega_{0}^{2}}
$$

i.e., the jitter gives an additional term to the CRB depending only on the jitter noise variance and the number of estimation data.

- Multisine input With a multisine input signal

$$
u(t)=\sum_{i=1}^{L} \alpha_{i} \sin \left(\omega_{i} t\right), \frac{1}{2} \sum_{i=1}^{L} \alpha_{i}^{2}=1
$$

we get the $\mathrm{CRB}$

$$
\operatorname{Var}\left(\hat{\Delta}_{t_{i}}\right) \gtrsim \frac{\sigma_{\text {jitter }}^{2}}{N}+\frac{2 \sigma_{e}^{2}}{N \sum_{l=1}^{L} \alpha_{l}^{2} \omega_{l}^{2}}
$$

Again the jitter gives an additional term to the CRB. The contribution from the jitter to the CRB is independent of the number of tones and hence the same as for the single sinusoidal case (24).

\section{Simulations}

To evaluate the performance of the time error estimation method, a time interleaved ADC system has been simulated. In Figure 7 the spectrum of the output signal is shown before and after correction with estimated time errors. Here the input signal is a single sinusoid. We can see here that, after correction, the time errors cannot be seen above the noise floor. The convergence rate is different for different input signals and different number of ADCs, but usually the
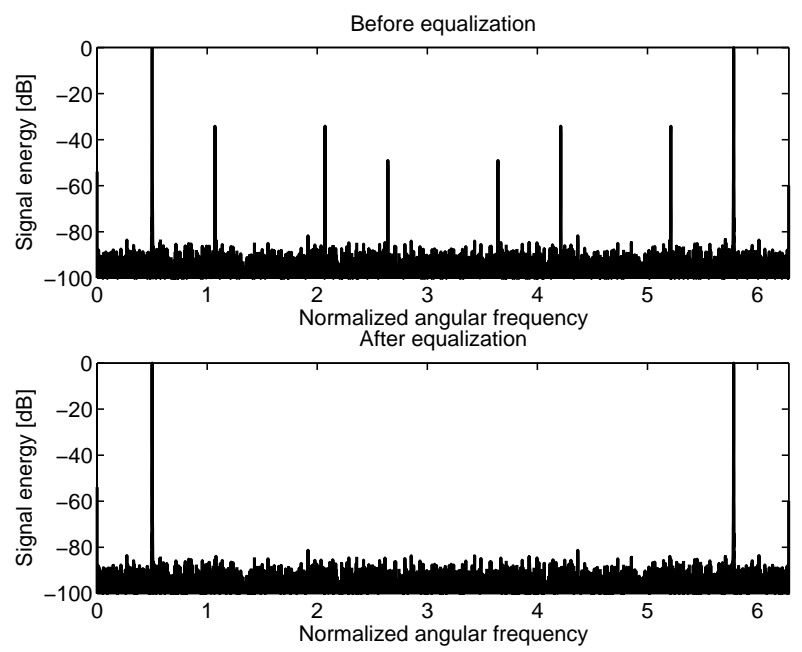

Fig. 7. Upper plot: The output spectrum of an interleaved ADC system with time errors. Lower plot: The same spectrum after compensation with estimated time error parameters. The parameters were estimated from $2^{14}$ samples per ADC.

parameters converge in about $10-50$ iterations. In Figure 8 an example of the convergence of the time error estimates is shown. The simulation is here done with four ADCs and sinusoidal input. The amount of data is here $2^{14}$ samples per batch. One iteration was done on each batch. In this example the parameters converge in about 20 iterations.

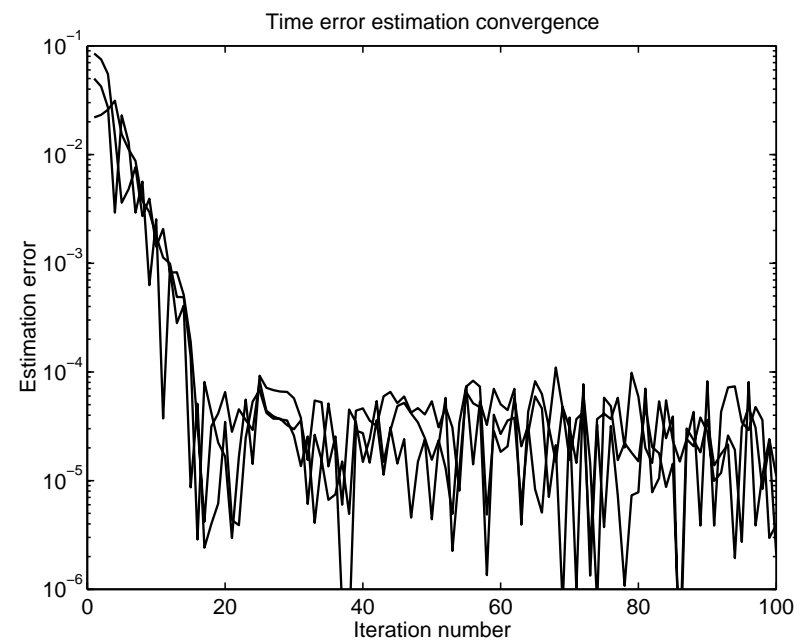

Fig. 8. Convergence of time error parameter estimates $\left(\hat{\Delta}_{t_{i}}-\Delta_{t_{i}}^{0}\right)$ for ADC system with four ADCs (three time errors). The estimation error is here shown in fractions of $T_{s}$.

To compare the estimation accuracy with the CRB the minimization has been done on one batch of data instead of updating with new data for each iteration. The estimation algorithm has been tested with different input signals and different signal parameters have been varied. One parameter at a time is changed according to the following list. The default value, used when other parameters are changed, is given inside parentheses. All frequencies in the simulations are normalized so that the foldover frequency $\omega_{f}=\pi$. 
- Sinusoidal input signal

- Angular frequency: $\omega_{0} \in[0.01,3.1]\left(\omega_{0}=1\right)$.

- Number of data per ADC: $N \in\left[2^{3}, 2^{16}\right]\left(N=2^{14}\right)$.

- Number of ADCs: $M \in[2,16](M=4)$.

- Quantization noise, given as number of bits: $n=$ $[2,16](n=10)$.

- Jitter variance: $\sigma_{\text {jitter }}^{2} \in[0,1]\left(\sigma_{\text {jitter }}^{2}=0\right)$.

- Multisine input signal

- Maximum angular frequency: $\omega_{0} \in[0.01,3.1]\left(\omega_{0}=\right.$ $1)$.

- Number of tones: $L \in[2,256](L=64)$.

- Low pass filtered white noise

- Cut off frequency: $\omega_{0} \in[0.01,3.1]$.

- Band pass filtered white noise, band width $10 \%$ of cut off frequency

- Cut off frequency: $\omega_{0} \in[0.01,3.1]$.

The true time error parameters have been generated randomly from a uniform distribution

$$
\begin{aligned}
& \text { For } i=1, \ldots, M-1 \\
& \Delta_{t_{i}}^{0} \in U\left[-0.1 T_{s}, 0.1 T_{s}\right]
\end{aligned}
$$

The standard deviations of the parameter estimation errors have been calculated from 25 Monte-Carlo simulations for each case in the list above. Some of the results from these simulations are shown in the plots described below. The results from simulations with the other paramters listed above are similar.

In Figure 9 the root mean square of the estimation error of the time error parameters is shown, as a function of the number of data, $N$. The input signal is here sinusoidal with input frequency $\omega_{0}=1$. For large values of $N$ the simulated parameter standard deviation is about a factor of 10 above the CRB. In Figure 10 the estimation error is shown with varying input signal frequency instead. We can see here that the estimation works well even close to the foldover frequency. For very low frequencies the input signal is very slowly varying. The output signal will therefore be constant for several samples due to the quantization. This means that much fewer samples contribute to the loss function and the performance is therefore worse. Figure 11 shows the estimation error as a function of the random jitter variance. We can see here that we get quite good estimates even when the jitter is in the same order of magnitude as the static time errors. In Figure 12 the estimation error is shown for a multisine input signal as a function of the maximum frequency. Figure 13 shows the estimation error with band limited white noise input. The pass band is here between $0.9 \omega_{c}$ and $\omega_{c}$ and the result is shown for varying $\omega_{c}$.

\section{MEASUREMENTS}

To validate the estimation method, the algorithm has also been tested on measured data from a time interleaved A/D converter system. The following parameters were used in the measurements

- 16 parallel ADCs with 12-bit precision.

- Sampling frequency, $f_{s}=5 \mathrm{MHz}$.

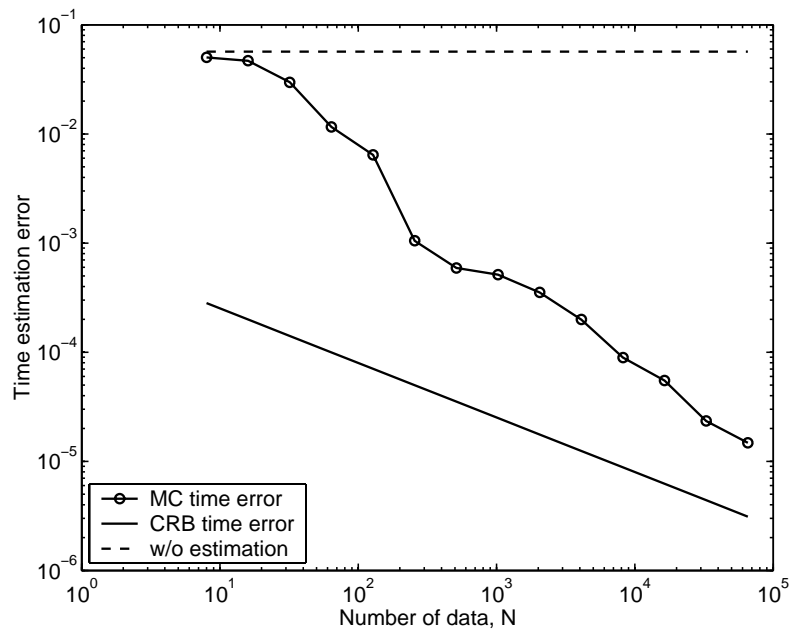

Fig. 9. Blind time estimation error as a function of the number of estimation data compared to the non-blind CRB. The input signal is here a single sinusoid with frequency $\omega_{0}=1$. The simulated values are calculated from 25 Monte Carlo simulations.

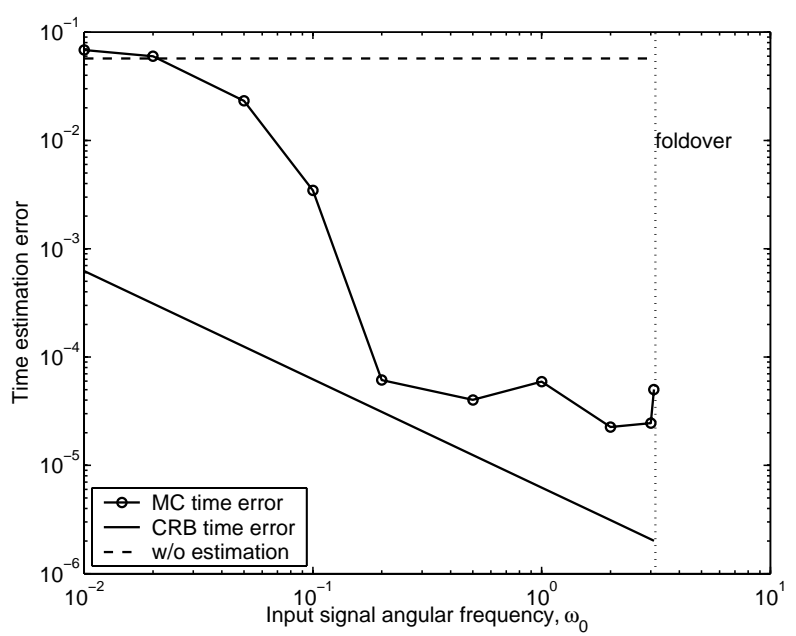

Fig. 10. Blind time estimation error as a function of input signal frequency compared to the non-blind CRB. The input signal is here a single sinusoid. The simulated values are calculated from 25 Monte Carlo simulations.

- Sinusoidal input signal with frequencies between $0.31 \mathrm{MHz}$ and $2.2 \mathrm{MHz}$.

- 8192 samples per ADC in each batch of data.

Here we have estimated the gain and offset errors also, as described in [9]. The signal generator is not perfect, which means that there is some harmonic distortion in the output spectrum. There are also other errors, besides the mismatch errors, that give distortion in the output signal. An example of an output spectrum is shown in Figure 14. Here we see that the mismatch distortion is small compared to the harmonic distortion. Therefore SFDR or SNDR [20] is not useful to measure the improvement after compensation for mismatch errors. Instead we study the improvement of the frequency components caused by the mismatch errors. In Figure 15 the same spectrum is shown after compensation with estimated mismatch parameters. The mismatch distortion is here no longer visible above the noise floor. To validate the mismatch 


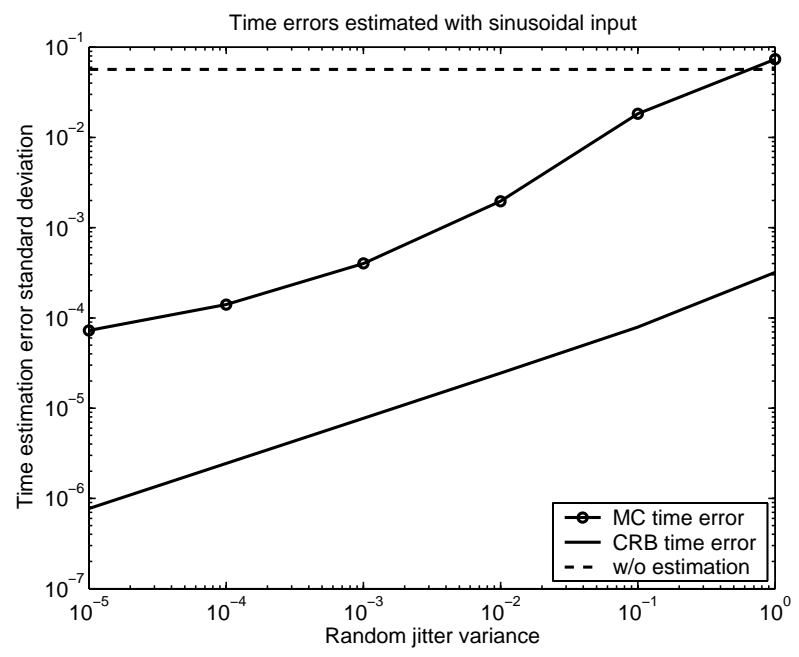

Fig. 11. Blind time estimation error as a function of the random jitter variance compared to the non-blind CRB. The input signal is here a single sinusoid with frequency $\omega_{0}=1$. The simulated values are calculated from 25 Monte Carlo simulations.

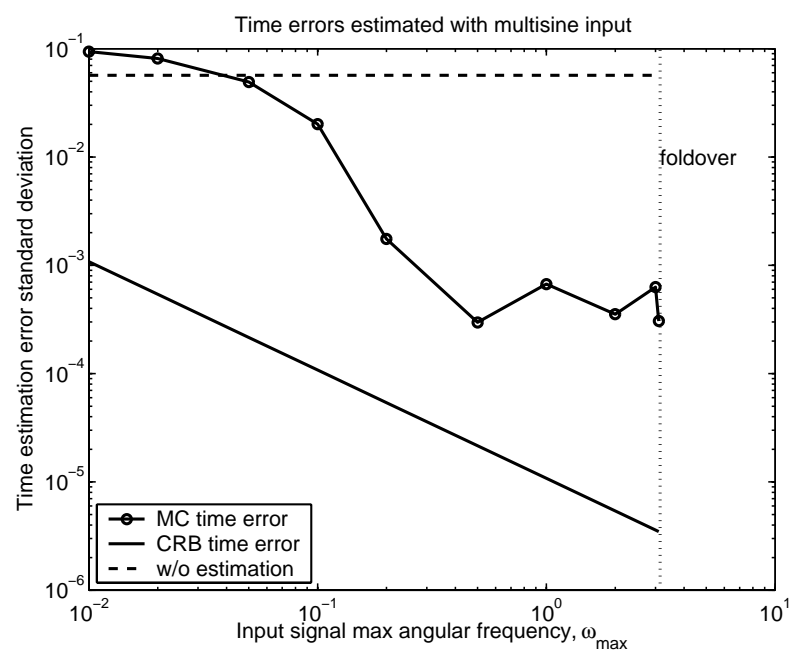

Fig. 12. Blind time estimation error as a function of input signal maximum frequency compared to the non-blind CRB. The input signal is here a multisine signal with 64 tones. The simulated values are calculated from 25 Monte Carlo simulations.

error estimation algorithm a parameter estimate was calculated for each input signal frequency and all signals were then compensated with each estimate. In Figure 16 the mean improvement of the gain and time error distortion components is shown. Since the sampling frequency is quite low, the time errors relative to the sampling interval are very small. This means that the time error distortion is very small, especially for low frequency signals, and therefore cannot be improved much. But we still see some improvement after the time error compensation.

\section{CONCLUSion}

A time interleaved ADC system is a good option to significantly increase the sampling rate of A/D conversion. However, due to errors in the manufacturing process, the

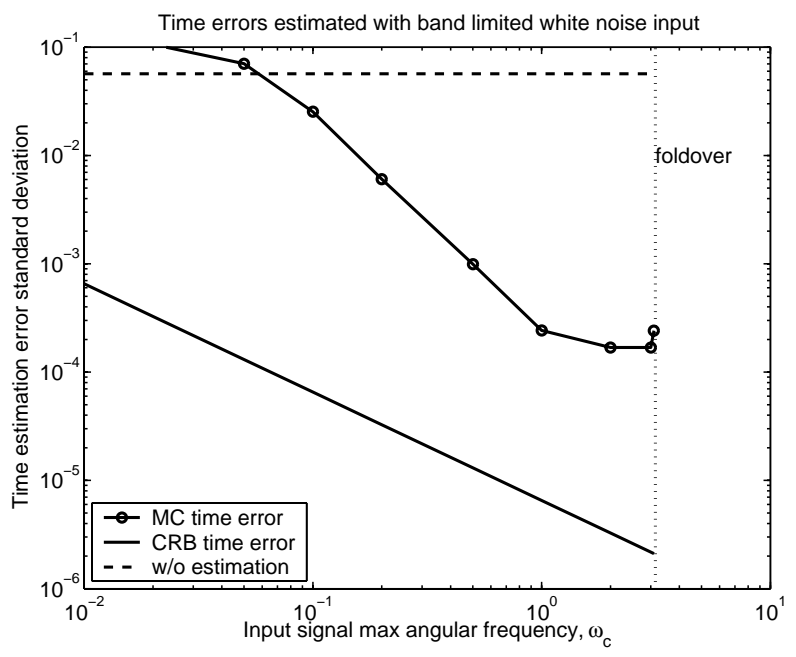

Fig. 13. Blind time estimation error as a function of input signal maximum frequency compared to the non-blind CRB. The input signal is here band limited white noise with pass band between $0.9 \omega_{c}$ and $\omega_{c}$. The simulated values are calculated from 25 Monte Carlo simulations.

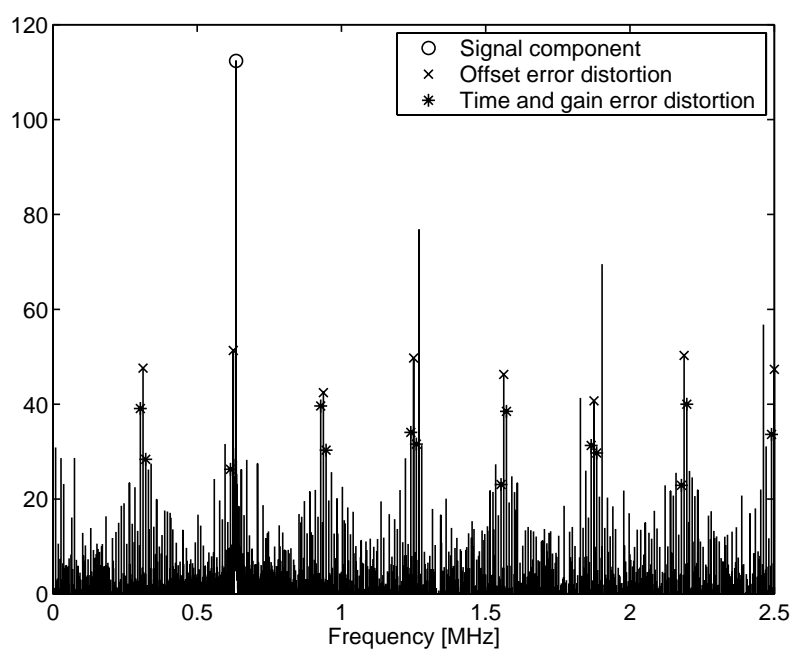

Fig. 14. Output spectrum from ADC measurement. The signal component is marked by 'o', the offset error distortion is marked by ' $\mathrm{x}$ ' and the gain error distortion is marked by ' $*$ '.

ADCs in the time interleaved system are not exactly identical. This means that mismatch errors in time, gain and offset are introduced. The mismatch errors cause distortion in the sampled signal. Calibration of ADCs is time consuming and costly. Further, the mismatch errors may change slowly, with for instance temperature and aging. Therefore it is preferable to continuously estimate the mismatch errors while the ADC is used.

In this paper, we have studied the time errors in a time interleaved ADC system. We have presented a method for estimation and compensation of the time mismatch errors. As opposed to other methods for estimation of time errors, this estimation method is blind, so that it does not require any special calibration signal or measurement of the input signal. It only requires that the input signal is band limited to the foldover frequency of the complete ADC system. The method is also 


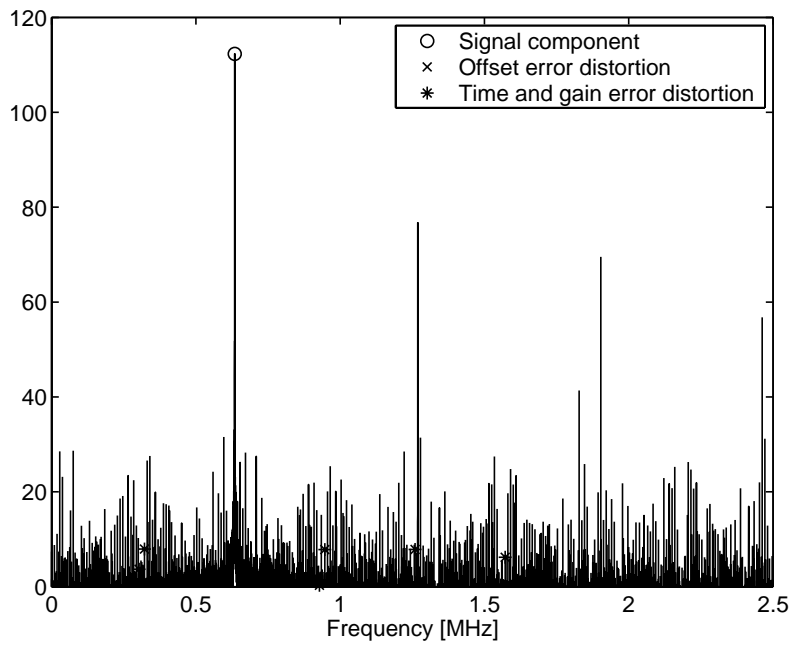

Fig. 15. Output spectrum from ADC measurement after compensation with estimated mismatch errors. Here the mismatch distortion is no longer visible above the noise floor.

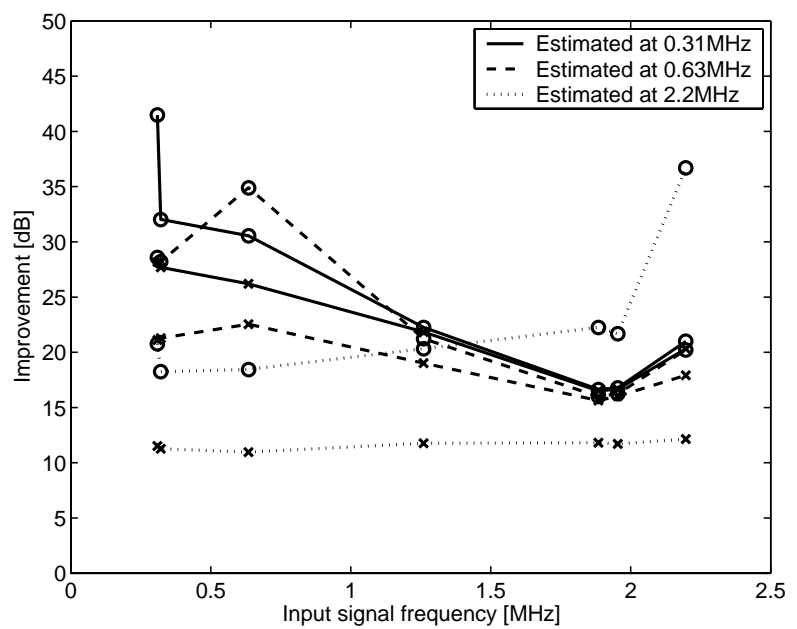

Fig. 16. Gain and time error distortion improvement. The improvement is shown for three sets of estimated parameters, estimated from sinusoidal signals with frequencies $0.31 \mathrm{MHz}, 0.63 \mathrm{MHz}$ and $2.2 \mathrm{MHz}$. The curves marked with ' $x$ ' show the improvement after compensation with only the gain error parameters and the curves marked with 'o' show the improvement after compensation with both gain and time error parameters.

adaptive, so the estimates are updated if the mismatch errors change slowly. The time error estimates that the estimation method generates are unbiased if the reconstruction changes the time error parameters linearly and simulations indicate the estimates are unbiased also when the real reconstruction is used. This means that the estimation accuracy can be made arbitrarily good by increasing the amount of estimation data.

We have also calculated the Cramer-Rao bound for the estimated parameters. The estimation accuracy from simulations does not reach the CRB, since the CRB is calculated assuming known input. However, the standard deviation of the estimated time errors decay with the amount of data at the same rate as the CRB.

The estimation method has also been verified on data from a real time interleaved ADC system. In a real ADC there

are other distortions, besides the mismatch error distortion. However, the measurement results show that the estimation method works well even if the ADCs are not ideal.

\section{REFERENCES}

[1] W. Black and D. Hodges, "Time interleaved converter arrays," IEEE Journal of Solid-State Circuits, vol. SC-15, no. 6, pp. 1022-1029, December 1980.

[2] Y.-C. Jenq, "Digital spectra of nonuniformly sampled signals: A robust sampling time offset estimation algorithm for ultra high-speed waveform digitizers using interleaving," IEEE Transactions on Instrumentation and Measurement, vol. 39, no. 1, pp. 71-75, February 1990.

[3] N. Kurosawa, K. Maruyama, H. Kobayashi, H. Sugawara, and K. Kobayashi, "Explicit formula for channel mismatch effects in timeinterleaved ADC systems," in Proc. IMTC, vol. 2, 2000, pp. 763-768.

[4] J. Corcoran, "Timing and amplitude error estimation for time-interleaved analog-to-digital converters," October 1992, US Patent nr. 5,294,926.

[5] H. Jin and E. Lee, "A digital-background calibration technique for minimizing timing-error effects in time-interleaved ADC's," IEEE Transactions on Cicuits and Systems, vol. 47, no. 7, pp. 603-613, July 2000.

[6] J. Elbornsson and J.-E. Eklund, "Blind estimation of timing errors in interleaved AD converters," in Proc. ICASSP 2001, vol. 6. IEEE, 2001, pp. 3913-3916.

[7] J. Elbornsson, K. Folkesson, and J.-E. Eklund, "Measurement verification of estimation method for time errors in a time-interleaved A/D converter system," in Proc. ISCAS 2002. IEEE, 2002.

[8] J.-E. Eklund and F. Gustafsson, "Digital offset compensation of timeinterleaved ADC using random chopper sampling," in IEEE International Symposium on Circuits and Systems, vol. 3, 2000, pp. 447-450.

[9] J. Elbornsson, F. Gustafsson, and J.-E. Eklund, "Blind adaptive equalization of mismatch errors in time interleaved A/D converter system," 2003, submitted to IEEE Transactions on Circuits and Systems.

[10] L. Ljung, System Identification, Theory for the user, 2nd ed. PrenticeHall, 1999.

[11] W. Gardner, Introduction to random processes with applications to signals and systems. New York: Macmillan Publishing Company, 1986.

[12] — Statistical spectral analysis, a nonprobabilistic theory. Englewood Cliffs, New Jersey: Prentice hall, 1988.

[13] M. Unser, "Splines - a perfect fit for signal and image processing," IEEE Signal Processing Magazine, pp. 22-38, November 1999.

[14] P. Löwenborg, "Asymmetric filter banks for mitigation of mismatch errors in high-speed analog-to-digital converters," Phd thesis 787, Department of Electrical Engineering, Linköping University, Linköping, Sweden, December 2002.

[15] A. Papoulis, Signal Analysis. McGraw-Hill, 1977.

[16] J. Elbornsson, "Analysis, estimation and compensation of mismatch effects in A/D converters," Phd thesis 811, Department of Electrical Engineering, Linköping University, Linköping, Sweden, May 2003.

[17] M. Hayes, Statistical digital signal processing and modeling. Wiley, 1996.

[18] J. Dennis and R. Schnabel, Numerical Methods for Unconstrained Optimization and Nonlinear Equations. Prentice-Hall, 1983.

[19] A. Gut, An Intermediate Course in Probability. Springer-Verlag, 1995.

[20] R. van de Plassche, Integrated Analog-to-Digital and Digital-to-Analog Converters. Kluwer Academic Publishers, 1994.

PLACE

PHOTO

HERE
Jonas Elbornsson Jonas Elbornsson received his M.Sc. degree in electrical engineering 1998 and the Ph.D. degree in Automatic Control 2003, both from Linköping University. In 2003 he joined MathCore Engineering $\mathrm{AB}$, where he works with modeling and simulation. 


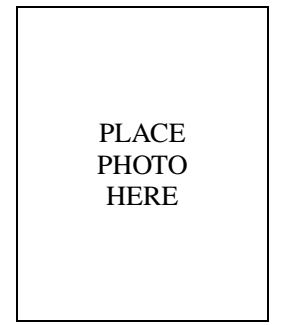

Fredrik Gustafsson Fredrik Gustafsson is professor in Communication Systems at the Department of Electrical Engineering, Linkping University, since 1999. He received the M.Sc. degree in electrical engineering 1988 and the Ph.D. degree in Automatic Control, 1992, both from Linkping University.

His research interests are in stochastic signal processing, adaptive filtering and change detection, with applications to communication, vehicular, airborne and audio systems.

$\mathrm{He}$ is the author of four books, over 100 international journal and conference papers and some 15 patent applications, and he is a co-founder of three companies in the signal processing area. He is an associate editor of IEEE Transactions on Signal Processing.

Jan-Erik Eklund Jan-Erik Eklund got his M.Sc. and $\mathrm{Ph}$. D. degrees from Linkping University, Linking, Sweden, in 1993 and 1997 respectively. In 1997 he joined Microelectronics Research Center at Ericsson Microelectronics AB, where he was involved in A/D converter research. Since 2002, he is with Infineon Technologies Wireless Solution Sweden AB. He hold several patents in the field of data conversion. 